\title{
Constraining the Beaming of Gamma-Ray Bursts with Radio Surveys
}

\section{Citation}

Perna, Rosalba, and Abraham Loeb. 1998. "Constraining the Beaming of Gamma-Ray Bursts with Radio Surveys." The Astrophysical Journal 509 (2): L85-88. https://doi.org/10.1086/311784.

\section{Permanent link}

http://nrs.harvard.edu/urn-3:HUL.InstRepos:41393210

\section{Terms of Use}

This article was downloaded from Harvard University's DASH repository, and is made available under the terms and conditions applicable to Other Posted Material, as set forth at http:// nrs.harvard.edu/urn-3:HUL.InstRepos:dash.current.terms-of-use\#LAA

\section{Share Your Story}

The Harvard community has made this article openly available.

Please share how this access benefits you. Submit a story.

\section{Accessibility}




\title{
CONSTRAINING THE BEAMING OF GAMMA-RAY BURSTS WITH RADIO SURVEYS
}

\author{
Rosalba Perna And Abraham Loeb \\ Harvard-Smithsonian Center for Astrophysics, 60 Garden Street, Cambridge, MA 02138 \\ Received 1998 October 6; accepted 1998 October 22; published 1998 November 10
}

\begin{abstract}
The degree of beaming in gamma-ray bursts (GRBs) is currently unknown. The uncertainty in the $\gamma$-ray-beaming angle, $\theta_{b}$, leaves the total energy release $\left(\propto \theta_{b}^{2}\right)$ and the event rate per galaxy $\left(\propto \theta_{b}^{-2}\right)$ unknown to within orders of magnitude. Since the delayed radio emission of GRB sources originates from a mildly relativistic shock and receives only weak relativistic beaming, the rate of radio-selected transients with no GRB counterparts can be used to set an upper limit on $\theta_{b}^{-2}$. We find that a VLA survey with a sensitivity of $\sim 0.1 \mathrm{mJy}$ at $10 \mathrm{GHz}$ could identify $\gtrsim 2 \times 10^{4}\left(\theta_{b} / 10^{\circ}\right)^{-2}$ radio afterglows across the sky if each source is sampled at least twice over a period of 1 month or longer. From the total number of $\gtrsim 0.1 \mathrm{mJy}$ sources observed at $8.44 \mathrm{GHz}$ and the fraction of fading sources at $1.44 \mathrm{GHz}$, we get the crude limit $\theta_{b} \gtrsim 6^{\circ}$.
\end{abstract}

Subject heading: gamma rays: bursts

\section{INTRODUCTION}

Gamma-ray burst (GRB) sources were discovered historically in $\gamma$-rays because they are rare and hence require continuous monitoring of large areas of the sky. The necessary all-sky monitoring program was first made feasible in the $\gamma$-ray regime, hence the name GRBs. By now, the prompt $\gamma$-ray emission is known to be followed in most cases by delayed emission in the X-ray (Costa et al. 1997), optical (van Paradijs et al. 1997; Fruchter et al. 1998a, 1998b), and radio (Frail 1998; Frail et al. 1998) bands. Given the recent discovery of afterglows, it is now timely to explore the feasibility of searching for GRB sources at longer wavelengths than traditionally attempted. The importance of complementary searches is highlighted by the possibility that some sources might be $\gamma$-ray faint for geometric or physical reasons. Such sources can only be found at long wavelengths, e.g., in radio surveys. Indeed, if the $\gamma$-ray emission is beamed, then there should be a population of radio afterglows that are $\gamma$-ray faint (Rhoads 1997). This follows from the fact that the bulk Lorentz factor of the emitting material in GRB sources declines with time and is only of order unity when the late radio emission takes place (Waxman, Kulkarni, \& Frail 1998), hence making the effect of relativistic beaming weak at that time.

The evolution of a jet resembles that of a spherical fireball (i.e., the jet behaves like a conical section of a spherical fireball), as long as the expansion Lorentz factor is larger than the inverse of the jet opening angle, $\Gamma>\theta_{b}^{-1}$. The smooth powerlaw decline of optical afterglows, observed over a timescale of days to months for GRB 970508 and GRB 970228 (Livio et al. 1997; Sokolov et al. 1998), suggests that these fireballs behaved as if they were spherically symmetric on angular scales ranging from $1 / \Gamma(1$ day $) \sim \frac{1}{8}$ to $1 / \Gamma(1$ month $) \sim \frac{1}{3}$ (Rhoads 1997, 1998; Waxman 1997a). However, there is still a missing gap in afterglow observations on the time window of minutes to several hours following a GRB. During this time, the fireball decelerates from $\Gamma \sim 100$ to $\Gamma \sim 10$, and so there is uncertainty about the structure of the fireball on angular scales of $\sim 0.01-0.1$ rad. It is still possible that the highly relativistic expansion with $\Gamma \sim 10^{2}$ is restricted only to a very small angular diameter, $\theta_{b} \sim 1 / \Gamma=0$. 6 . In fact, the popular GRB scenarios of binary coalescence of compact stars or failed supernovae favor strong collimation over spherical expansion (Woosley 1998; Mészá- ros, Rees, \& Wijers 1998). The search for long-wavelength transients with no GRB counterparts can set important constraints on $\theta_{b}$ and hence on these models.

In this Letter, we show that a $0.1 \mathrm{mJy}$ radio survey at the VLA $^{1}$ (analogous to the FIRST survey; Helfand et al. 1996) can provide strong constraints on $\theta_{b}$. The surveyed sources need to be sampled at least twice over a timescale on the order of a month or longer, since short-term variability may also be caused by scintillations of steady pointlike sources. In our calculations, we assume that the GRB rate is proportional to the cosmic star formation rate and neglect the small fraction ( $\$ 10 \%)$ of GRBs that might be related to local radio supernovae (Bloom et al. 1998). We model the time-dependent luminosity of the radio afterglows based on observational data and allow for a scatter in their peak luminosities. Our model is described in detail in $\S 2$. The number count of radio afterglows that it predicts for radio surveys is calculated in $\S 3$. By comparing this number with that of fading sources in existing radio surveys, we get a lower limit on $\theta_{b}$. Finally, our main conclusions are summarized in $\S 4$.

\section{STATISTICS OF RADIO GRB AFTERGLOWS}

The isotropy of GRBs (Meegan et al. 1993; Briggs et al. 1993) and the flattening of their number count distribution at faint fluxes suggest that most GRBs occur at cosmological distances. This hypothesis has been confirmed by the detection of Fe II and $\mathrm{Mg}$ II absorption lines at a redshift of $z=0.835$ in the optical spectrum of GRB 970508 (Metzger et al. 1997), the inference of a redshift $z=3.42$ for the host galaxy of GRB 971214 (Kulkarni et al. 1998), and the detection of emission and absorption lines at $z=0.966$ for the host of GRB 980703 (Djorgovski et al. 1998).

The energy scale of cosmological GRBs, $\sim 10^{53} f_{b}$ ergs (Kulkarni et al. 1998), corresponds to the rest mass energy of a fraction of a solar mass and implies a link between their energy budget and compact stars. Indeed, the most popular models for the origin of GRBs relate them to compact stellar remnants,

\footnotetext{
${ }^{1}$ The VLA is a facility of the National Radio Astronomy Observatory, which is operated by Associated Universities, Inc., under contract with the National Science Foundation.
} 
such as neutron stars or black holes (see, e.g., Eichler et al. 1989; Narayan, Paczyński, \& Piran 1992; Usov 1992; Woosley 1993; Mészáros et al. 1998). Since these remnants form out of massive stars not long after their birth, it is reasonable to assume that the GRB rate traces the star formation rate without a significant delay. This scenario was investigated by Wijers et al. (1997), who derived a best-fit constant of proportionality between the GRB occurrence rate, $R(z)$, and the star formation rate per comoving volume, $\rho(z)$, based on the requirement that the former fits the observed number count distribution of GRBs. The cosmic star formation rate as a function of redshift has been calibrated based on observations of the $U$ - and $B$-band luminosity density evolution in the Hubble Deep Field (Madau et al. 1996; Madau, Pozzetti, \& Dickinson 1998). The fit made by Wijers et al. (1997) yields a local GRB rate of $R(z=$ $0)=(0.14 \pm 0.02) \times 10^{-9} \mathrm{Mpc}^{-3} \mathrm{yr}^{-1}$ in an $\Omega=1, \Lambda=0$, and $H_{0}=70 \mathrm{~km} \mathrm{~s}^{-1} \mathrm{Mpc}^{-1}$ cosmology.

We model the frequency and time dependence of the afterglow luminosity, $L_{\nu}(t)$, using the simplest unbeamed synchrotron model (see, e.g., Waxman 1997a, 1997b), with a luminosity per unit emitted frequency $\tilde{\nu}$ of

$$
L_{\tilde{\nu}}(t)=L_{\nu_{m}}\left[\frac{\tilde{\nu}}{\nu_{m}(t)}\right]^{-\alpha}
$$

where $\nu_{m}(t)=8.8 \times 10^{2}(1+z)^{1 / 2}(t / \text { month })^{-3 / 2} \mathrm{GHz}, t$ is time at the source frame, and $z$ is the source redshift. The observed frequency $\nu$ is related to the emitted frequency $\tilde{\nu}$ through $\nu=\tilde{\nu} /(1+z)$. The spectral index $\alpha$ is chosen to have the values $\alpha_{1}=\frac{1}{3}$ for $\nu \leq \nu_{m}$ and $\alpha_{2}=0.7$ for $\nu>\nu_{m}$, so as to match the temporal decay slope observed for GRB 970228 (Fruchter et al. 1998b) and GRB 970508 (Galama et al. 1998).

We consider a population of afterglow sources characterized by a total comoving rate per unit volume, $R(z)$, and by a peak flux $F_{\nu_{m}}\left(z, L_{\nu_{m}}\right)=L_{\nu_{m}}(1+z) / 4 \pi D_{L}^{2}(z)$, where $D_{L}(z)$ is the cosmology-dependent luminosity distance. For consistency with the numbers derived by Wijers et al. (1997), we assume $\Omega=$ $1, \Lambda=0, h=0.7$, and $D_{L}(z)=\left(2 c / H_{0}\right)(1+z-\sqrt{1+z})$. Note that if the radio flux of GRB sources is strictly proportional to their $\gamma$-ray flux, then our number count results are independent of the choice of cosmological parameters to zeroth order; this follows from the fact that Wijers et al. (1997) calibrate their results based on GRB number count data.

We allow for a spread in the peak luminosity $L_{\nu_{m}}$ of the afterglows by using a lognormal probability distribution (and thus minimizing the number of free parameters),

$$
P\left(L_{\nu_{m}}\right) d L_{\nu_{m}}=\frac{1}{\sqrt{2 \pi \sigma^{2}}} \exp \left\{-\frac{\left[\ln \left(L_{\nu_{m}}\right)-\ln \left(L_{\star}\right)\right]^{2}}{2 \sigma^{2}}\right\} \frac{d L_{\nu_{m}}}{L_{\nu_{m}}}
$$

The mean of this distribution equals $\left\langle L_{\nu_{m}}\right\rangle=L_{\star} \exp \left(\sigma^{2} / 2\right)$. We normalize its value by matching the mean peak flux,

$$
\left\langle F_{\nu_{m}}\right\rangle=\frac{\int_{0}^{\infty} d L_{\nu_{m}} P\left(L_{\nu_{m}}\right) \int_{0}^{\infty} d z[R(z) /(1+z)] F_{\nu_{m}}\left(z, L_{\nu_{m}}\right) d V_{c}}{\int_{0}^{\infty} d L_{\nu_{m}} P\left(L_{\nu_{m}}\right) \int_{0}^{\infty} d z[R(z) /(1+z)] d V_{c}},
$$

to its observed value in radio afterglows. The observed 8.44 $\mathrm{GHz}$ light curves of GRB 970508, GRB 980329, and GRB
980703 yield $\left\langle F_{\nu_{m}}\right\rangle \approx 0.7 \mathrm{mJy}$. For the above cosmology, this implies $\left\langle L_{\nu_{m}}\right\rangle \approx 1.5 \times 10^{31} h_{70}^{-2}$ ergs s${ }^{-1} \mathrm{~Hz}^{-1}$.

The calculation of the afterglow number counts should also incorporate the fact that not all GRBs have afterglows at radio frequencies. The fraction $f_{\text {radio }}$ of radio-loud GRBs is still uncertain, but the observation that four out of $\sim 15-17$ welllocalized GRBs (namely, GRB 970508, GRB 980329, GRB 980519, and GRB 980703) were detected in the radio (D. Frail 1998 , private communication) implies that $f_{\text {radio }} \simeq 25 \%$. Also, the afterglow radio flux might have a break in its power-law evolution and decay more rapidly than predicted by equation (1) after a particular time (e.g., during the nonrelativistic phase of the shock hydrodynamics). We artificially introduce a free parameter, $t_{\text {cutoff }}$, after which we set the emitted radio flux to zero. Current data do not allow an empirical calibration of $t_{\text {cutoff }}$, and so we leave it as a free parameter. We assume that $t_{\text {cutoff }} \gtrsim 3$ months since a simple power-law decline was observed in GRB 970508 for over 3 months (Waxman et al. 1998).

The late phase of the radio emission, several months after a GRB event, originates from semirelativistic material with $\Gamma \sim 1$ and receives only weak relativistic beaming. However, the $\gamma$-ray emission is emitted by material with $\Gamma \gtrsim 10^{2}$ and could be confined to a cone with an angular diameter $\theta_{b} \ll 1$. In this case, the rate of radio afterglows would be enhanced by a factor $f_{b}^{-1}=4 \pi / \pi\left(\theta_{b} / 2\right)^{2}=5.25 \times 10^{2}\left(\theta_{b} / 10^{\circ}\right)^{-2}$, relative to the unbeamed case.

Typical radio surveys do not provide continuous monitoring of the sky, but rather they provide a sequence of "snapshots" of each resolution element on the sky. In observations where the minimum detectable flux of a source is $F_{\nu}$, the total number of events across the sky that are brighter than $F_{\nu}$ at observed frequency $\nu$ is given by

$$
N\left(>F_{\nu} ; \nu\right)=f_{b}^{-1} f_{\text {radio }} \int_{0}^{\infty} d L_{\nu_{m}} P\left(L_{\nu_{m}}\right) N^{\prime}\left(>F_{\nu} ; \nu, L_{\nu_{m}}\right),
$$

where

$$
N^{\prime}\left(>F_{\nu} ; \nu, L_{\nu_{m}}\right)=\int_{0}^{z_{\mathrm{lim}}\left(L_{\nu_{m}}\right)} R(z) t_{*}\left(z, F_{\nu}, \nu, L_{\nu_{m}}\right) \frac{d V_{c}}{d z} d z
$$

Here $d V_{c}=4\left(c / H_{0}\right)^{3}(1+z-\sqrt{1+z})^{2}(1+z)^{-7 / 2} d \Omega d z$ is the comoving volume element within a solid angle $d \Omega$ and redshift interval $d z$, and $z_{\text {lim }}\left(L_{\nu_{m}}\right)$ is found from the algebraic relation $F_{\nu_{m}}\left(z_{\text {lim }}, L_{\nu_{m}}\right)=F_{\nu}$. The time $t_{*}\left(z, F_{\nu}, \nu\right)$ represents the duration over which an event at a redshift $z$ is brighter than the limiting flux $F_{\nu}$ at the observed frequency $\nu$,

$$
\begin{aligned}
& t_{*}\left(z, F_{\nu}, \nu, L_{\nu_{m}}\right)=\min \left(t_{\text {cutoff }},\left[\frac{(1+z) \nu}{\nu_{m_{0}}}\right]^{-2 / 3}\right. \\
& \left.\quad \times\left\{\left[\frac{F_{\nu}}{F_{\nu_{m}}\left(z, L_{\nu_{m}}\right)}\right]^{-2 / 3 \alpha_{2}}-\left[\frac{F_{\nu}}{F_{\nu_{m}}\left(z, L_{\nu_{m}}\right)}\right]^{-2 / 3 \alpha_{1}}\right\}\right),
\end{aligned}
$$




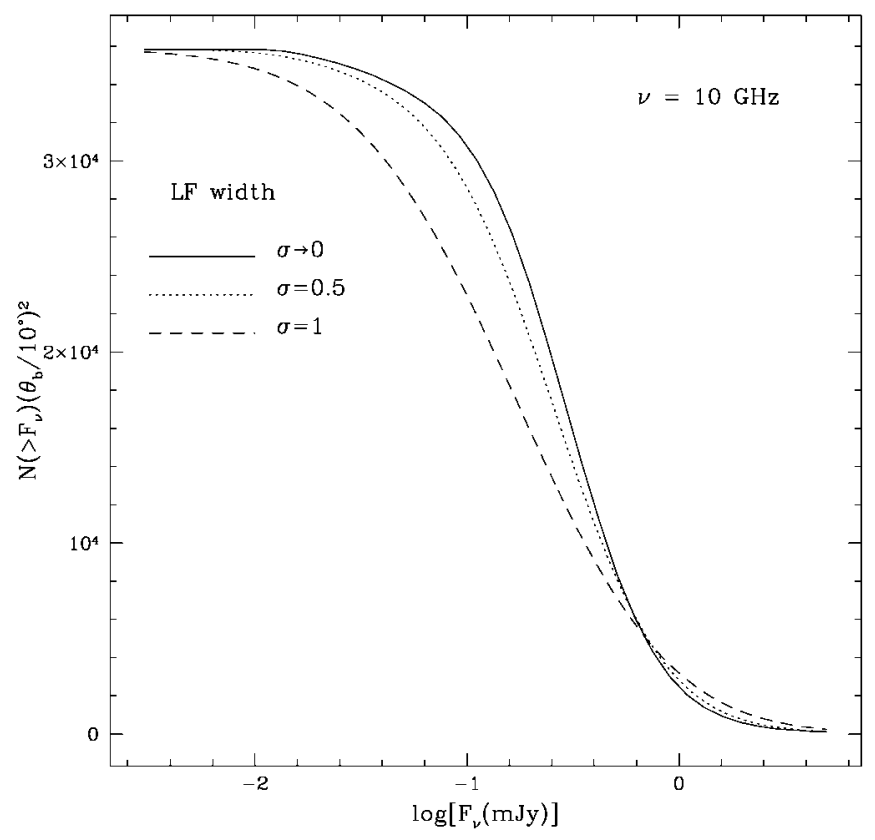

FIG. 1.-Number of radio-loud afterglows that would be detected in an allsky radio survey as a function of its flux detection threshold $F_{\nu}$ at $10 \mathrm{GHz}$. The different lines correspond to different values of the width of the GRB luminosity function, $\sigma$ (see eq. [2]). The fraction of the radio-loud afterglows is assumed to be $f_{\text {radio }}=25 \%$, and the afterglow emission is truncated after a time $t_{\text {cutoff }}=6$ months in all sources. The normalization factor of the number counts involves the opening angle of the $\gamma$-ray emission, $\theta_{b}$, in units of $10^{\circ}$.

\section{CONSTRAINTS FROM RADIO SURVEYS}

The VLA FIRST survey monitored $1550^{\circ}$ of sky with a sensitivity of $1 \mathrm{mJy}$ at a frequency of $1.5 \mathrm{GHz}$ (Helfand et al. 1996). The intervals between the observations of each source ranged between 3 minutes and 3 weeks. Unfortunately, the properties of this survey were not optimal for the purpose of identifying GRB afterglows. Here we consider a hypothetical survey that is optimized for this purpose. Such a survey would use a higher frequency in order to avoid the suppression of the afterglow flux by synchrotron self-absorption at the source (typically observed at $\left.\$ 5[(1+z) / 2]^{-1} \mathrm{GHz}\right)$. Moreover, since short-term variability could also be caused by scintillations, the surveyed sources should be monitored at least twice over an extended period on the order of a month or more. After a few months, all the radio-loud afterglows would fade significantly and thus be easily distinguishable from other variable source populations. The variability level of $\sim 0.1-1 \mathrm{mJy}$ sources can be determined accurately, given the high sensitivity of the VLA.

Figure 1 shows the number of radio afterglows that are expected to be found in an all-sky survey at $10 \mathrm{GHz}$, as a function of its flux threshold. The plotted number depends on a normalization factor involving the $\gamma$-ray-beaming angle $\theta_{b}$ in units of $10^{\circ}$. We assume $f_{\text {radio }}=25 \%$ and show results for different values of $\sigma$ while keeping $t_{\text {cutoff }}$ fixed at 6 months. Figure 2 shows the same for $\sigma \rightarrow 0$, but for different values of $t_{\text {cutoff }}$. Typically, a $0.1 \mathrm{mJy}$ survey similar to FIRST but at $10 \mathrm{GHz}$ would identify $\gtrsim 2 \times 10^{4}\left(\theta_{b} / 10^{\circ}\right)^{-2}$ afterglows across the sky if the $\gamma$-ray emission from GRBs is collimated to within an opening angle $\theta_{b}$.

Lower limits on the value of $\theta_{b}$ can already be placed by existing radio surveys. The total number of radio sources observed on the sky provides a robust upper limit on the number

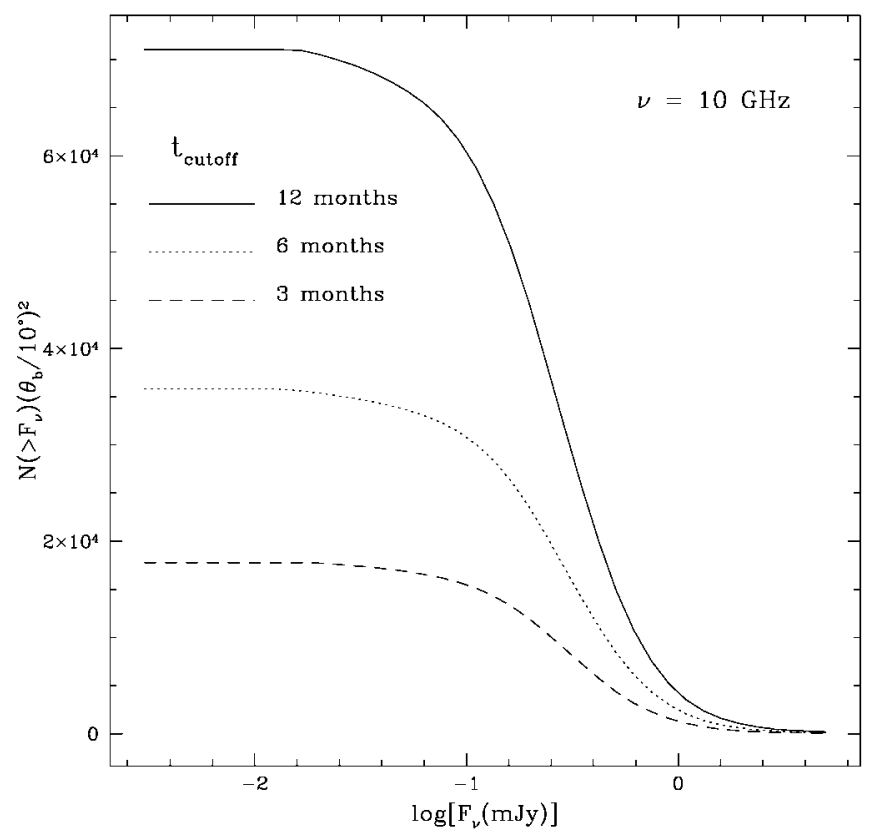

Fig. 2.- Same as in Fig. 1, but with a fixed $\sigma \rightarrow 0$ and different values of $t_{\text {cutoff }}$.

of GRB afterglows. This limit can be improved by considering only those sources that are unresolved and, at the same time, variable over a timescale of a month or longer. Still tighter constraints can be obtained by considering only the subset of all unidentified sources that, after a sufficiently long time $(\gtrsim 6$ months), fade away below the detection threshold. From a deep VLA survey, Windhorst et al. (1993) derived a maximum likelihood fit to the source number counts at $8.44 \mathrm{GHz}$ in the flux range of $14.5 \mu \mathrm{Jy}-1.5 \mathrm{mJy}$. By comparing a Westerbork and a VLA survey of the same field at $1.4 \mathrm{GHz}$, Oort \& Windhorst (1985) found that about $10 \%$ of the sources were variable on a timescale of a year, and only $\sim 3 \%$ were detected in one survey but not in the other. Thus, only $\sim 3 \%$ of the sources in this survey qualify as candidates for GRB afterglows. The submillijansky sources were typically found to be unresolved. Better angular resolution could, in principle, limit further the number of afterglow candidates by resolving other source populations.

Figure 3 shows the ratio between the predicted number of GRB afterglows $N\left(>F_{\nu}\right)$ and the total observed number of radio sources $N_{\text {obs }}$ (Windhorst et al. 1993) at $8.44 \mathrm{GHz}$. We denote the fraction of variable sources that fade away after a year by $f_{\text {fade }}$ and the fraction of pointlike (unresolved) sources by $f_{\text {point }}$. Based on the observed abundance of unresolved variable sub-millijansky sources at $1.4 \mathrm{GHz}$ (Oort \& Windhorst 1985), we set $f_{\text {fade }} f_{\text {point }}=3 \%$. The ratio between the predicted afterglow counts and the observed radio counts peaks at around 0.4-0.6 mJy. From the requirement that this ratio be smaller than unity, we get the lower limit $\theta_{b} \gtrsim 6^{\circ}$. This limit is based on the variability statistics of only $\sim 10^{2}$ sources (Oort \& Windhorst 1985) and hence suffers from large statistical uncertainties $\left(\min \left\{\theta_{b}\right\} \propto f_{\text {fade }}^{-1 / 2}\right.$ ). Surveys with a larger coverage of the sky are necessary in order to firm up the statistical significance of this limit.

\section{CONCLUSIONS}

We have found that a $0.1 \mathrm{mJy}$ all-sky survey at $10 \mathrm{GHz}$ should identify $\sim 2 \times 10^{4}\left(\theta_{b} / 10^{\circ}\right)^{-2} \mathrm{GRB}$ afterglows and there- 


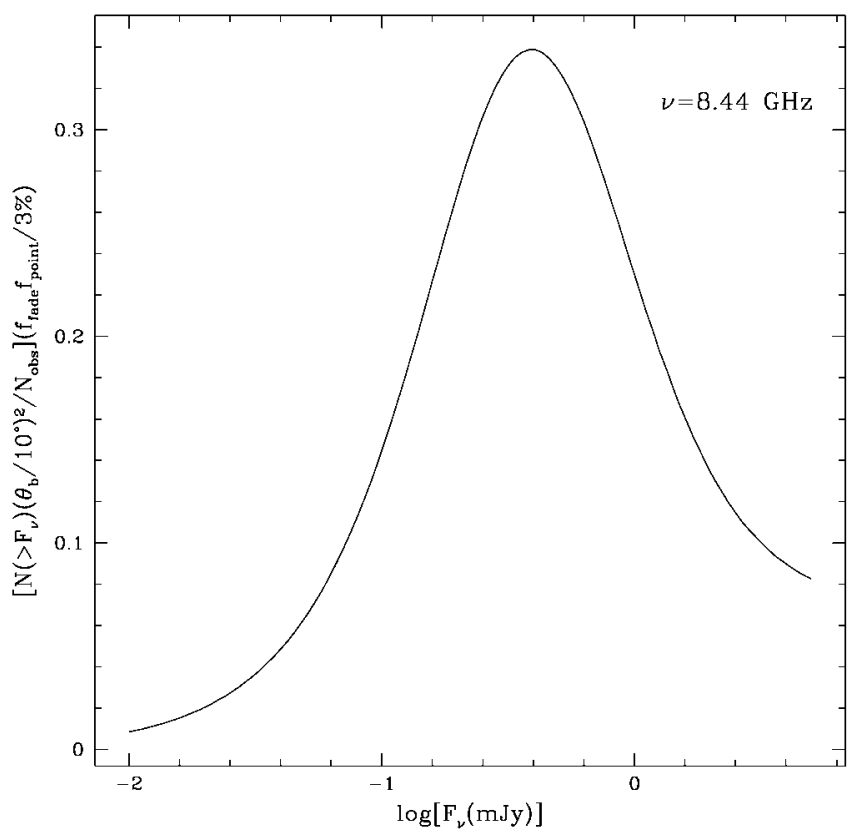

FIG. 3.-The ratio between the predicted cumulative number of GRB afterglows $N\left(>F_{\nu}\right)$ and the observed cumulative number of radio sources $N_{\text {obs }}$ (Windhorst et al. 1993), as a function of the flux threshold $F_{v}$ at $8.44 \mathrm{GHz}$. The observed number is uncertain by $\pm 40 \%$. The fraction of unresolved fading sources in the observed population is assumed to be $f_{\text {tade }} f_{\text {point }}=3 \%$, based on the $1.4 \mathrm{GHz}$ data (Oort \& Windhorst 1985). Model parameters are chosen as in Fig. 1, with $\sigma \rightarrow 0$. The condition that the plotted ratio be less than unity at any flux threshold sets a lower limit on the $\gamma$-ray-beaming angle $\theta_{b} \gtrsim 6^{\circ}$. fore could place important constraints on the GRB beaming angle, $\theta_{b}$. In fact, the total number of $\gtrsim 0.1 \mathrm{mJy}$ sources on the sky at $8.44 \mathrm{GHz}$ (Windhorst et al. 1993) and the fraction of fading unresolved sources at $1.4 \mathrm{GHz}$ (Oort \& Windhorst 1985) already yield the crude lower limit $\theta_{b} \gtrsim 6^{\circ}$. This result is only weakly sensitive to the width of the afterglow luminosity function (see Fig. 1) and is mainly uncertain because of the limited size of the sample of sub-millijansky sources that had been monitored for long-term variability (Oort \& Windhorst 1985). Nevertheless, the derived constraint is interesting, given the fact that the $\gamma$-ray emission in GRB sources originates from material with a bulk Lorentz factor $\Gamma \geqslant 10^{2}$ and could have been collimated to within an angle $\theta_{b} \sim 1 / \Gamma \lesssim 0.6$, which is narrower by more than an order of magnitude than our limit. In comparison, radio jets in quasars are collimated to within $\sim 15^{\circ}$ and possess Lorentz factors $\Gamma \lesssim 10$ (Begelman, Blandford, \& Rees 1984).

A future VLA survey, optimized to search for radio afterglows by monitoring sub-millijansky source variability at $\sim 10$ $\mathrm{GHz}$ over a timescale of several weeks to several months, could improve the above upper limit on $\theta_{b}$ considerably. Alternatively, such a search might identify a new class of radio afterglow events that fade away after a few months and have no GRB counterparts.

We thank Dale Frail for useful discussions. This work was supported in part by the NASA grant NAG5-7039.

\section{REFERENCES}

Begelman, M. C., Blandford, R. D., \& Rees, M. J. 1984, Rev. Mod. Phys., $56.2,255$

Bloom, J. S., Kulkarni, S. R., Harrison, F., Prince, T., \& Phinney, E. S. 1998, ApJ, 506, L105

Briggs, M. S., et al. 1993, in Proc. Huntsville Gamma-Ray Burst Workshop, ed. G. Fishman, J. Brainerd, \& K. Hurley (New York: AIP), 44

Costa, E., et al. 1997, Nature, 387, 783

Djorgovski, S. G., Kulkarni, S. R., Bloom, J. S., Goodrich, R., Frail, D. A., Piro, L., \& Palazzi, E. 1998, ApJ, 508, L17

Eichler, D., Livio, M., Piran, T., \& Schramm, D. N. 1989, Nature, 340, 126

Frail, D. A. 1998, in AIP Conf. Proc. 428, Gamma Ray Bursts, Fourth Huntsville Symp, ed. C. A. Meegan, R. D. Preece, \& T. M. Koshut (New York: AIP), 563

Frail, D. A., et al. 1998, GCN Circ. 128

Fruchter, A. S., et al. 1998a, ApJ, submitted (astro-ph/9807295)

. 1998b, in Proc. Fourth Huntsville Symp., Gamma-Ray Bursts, ed. C. A. Meegan, R. Preece, \& T. Koshut (New York: AIP), in press (astro-ph/9801169)

Galama, T. J., et al. 1998, ApJ, 500, L101

Helfand, D. J., Das, S. R., Becker, R. H., White, R. L., \& McMahon, R. G. 1996, in ASP Conf. Ser. 110, Blazar Continuum Variability, ed. H. R. Miller, J. R. Webb, \& J. C. Noble (San Francisco: ASP), 214

Kulkarni, S. R., et al. 1998, Nature, 393, 35

Livio, M., et al. 1997, ApJ, 489, L127

Madau, P., Ferguson, H. C., Dickinson, M. E., Giavalisco, M., Steidel, C. C., \& Fruchter, A. 1996, MNRAS, 283, 1388
Madau, P., Pozzetti, L., \& Dickinson, M. E. 1998, ApJ, 498, 106

Meegan, C. A., et al. 1993, in Proc. Huntsville Gamma-Ray Burst Workshop, ed. G. Fishman, J. Brainerd, \& K. Hurley (New York: AIP), 3

Mészáros, P., Rees, M. J., \& Wijers, R. A. M. J. 1998, NewA, submitted (astro-ph/9808106)

Metzger, R., et al. 1997, Nature, 387, 878

Narayan, R., Paczyński, B., \& Piran, T. 1992, ApJ, 395, L83

Oort, M. J., \& Windhorst, R. A. 1985, A\&A, 145, 405

Rhoads, J. E. 1997, ApJ, 487, L1

—. 1998, Proc. Fourth Huntsville Symp., Gamma-Ray Bursts, ed. C. A Meegan, R. Preece, \& T. Koshut (New York: AIP), in press (astro-ph/ 9712042)

Sokolov, V. V., et al. 1998, A\&A, 334, 177

Usov, V. V. 1992, Nature, 357, 472

van Paradijs, J., et al. 1997, Nature, 386, 686

Waxman, E. 1997a, ApJ, 485, L5

$$
\text { 1997b, ApJ, 489, L33 }
$$

Waxman, E. Kulkarni, S. R., \& Frail, D. A. 1998, ApJ, 497, 288

Wijers, R. A. M. J., Bloom, J. S., Bagla, J. S., \& Nataraja, P. 1997, MNRAS, 294, L13

Windhorst, R. A., Fomalont, E. B., Partridge, R. B., \& Lowenthal, J. D. 1993, ApJ, 405, 498

Woosley, S. E. 1993, ApJ, 405, 273 . 1998, ApJ, 405, 273 